\title{
Measure-valued solutions to the complete Euler system
}

\author{
By Jan BřEzINA and Eduard FeIREISL
}

(Received Feb. 15, 2017)

\begin{abstract}
We introduce the concept of dissipative measure-valued solution to the complete Euler system describing the motion of an inviscid compressible fluid. These solutions are characterized by a parameterized (Young) measure and a dissipation defect in the total energy balance. The dissipation defect dominates the concentration errors in the equations satisfied by the Young measure. A dissipative measure-valued solution can be seen as the most general concept of solution to the Euler system retaining its structural stability. In particular, we show that a dissipative measure-valued solution necessarily coincides with a classical one on its life span provided they share the same initial data.
\end{abstract}

\section{Introduction.}

In his pioneering work [10], DiPerna proposed a new concept of solution, known as measure-valued solution, to nonlinear systems of partial differential equations admitting uncontrollable oscillations. In particular with focus on the compressible Euler system and other related models of inviscid fluids. Later on, a similar strategy has been adopted even to problems involving viscous fluid flows, where compactness of the solution set is either absent or out of reach of the available mathematical tools, see e.g. the monograph Nečas et al. [20] and the references therein. Although existence of a measure-valued solution to a given problem is usually an almost straightforward consequence of a priori bounds, its uniqueness in terms of the initial data can be seen as the weakest point of this approach. In addition, the recent results of DeLellis, Székelyhidi and their collaborators $[\mathbf{7}],[\mathbf{8}],[\mathbf{9}]$ show that uniqueness may be in fact violated even within the class of more conventional weak solutions satisfying the standard entropy admissibility criteria.

Brenier et al. [5] proposed a new approach seeing the measure-valued solutions as possibly the largest class in which the family of smooth (classical) solutions is stable. In particular, they show the so-called weak (measure-valued)-strong uniqueness principle for the incompressible Euler system. Specifically, a classical and a measure-valued solution emanating from the same initial data coincide as long as the former exists. These results have been extended to the isentropic Euler and Navier-Stokes systems by Gwiazda et al. $[\mathbf{1 8}]$ and $[\mathbf{1 1}]$. The recently renewed interest in measure-valued solutions in fluid

2010 Mathematics Subject Classification. Primary 35L45; Secondary 35Q35, 76N15.

Key Words and Phrases. Euler system, measure-valued solution, weak-strong uniqueness, perfect gas.

The second author leading to these results has received funding from the European Research Council under the European Union's Seventh Framework Programme (FP7/2007-2013)/ ERC Grant Agreement 320078. The Institute of Mathematics of the Academy of Sciences of the Czech Republic is supported by RVO:67985840. 
mechanics has been also initiated by certain numerical experiments with oscillatory solutions, see Fjordholm et al. [15], [16], [17]. Following the philosophy of Brenier et al. [5], we focus on the concept of measure-valued solutions in the widest possible sense. Accordingly, using the fundamental laws of thermodynamics, we extract the minimal piece of information to be retained to preserve the weak-strong uniqueness principle.

We consider the complete Euler system describing the time evolution of the mass density $\varrho=\varrho(t, x)$, the velocity $\boldsymbol{u}=\boldsymbol{u}(t, x)$, and the (absolute) temperature $\vartheta=\vartheta(t, x)$ of a compressible inviscid fluid:

$$
\begin{aligned}
& \partial_{t} \varrho+\operatorname{div}_{x}(\varrho \boldsymbol{u})=0, \\
& \partial_{t}(\varrho \boldsymbol{u})+\operatorname{div}_{x}(\varrho \boldsymbol{u} \otimes \boldsymbol{u})+\nabla_{x} p(\varrho, \vartheta)=0, \\
& \partial_{t}\left(\frac{1}{2} \varrho|\boldsymbol{u}|^{2}+\varrho e(\varrho, \vartheta)\right)+\operatorname{div}_{x}\left[\left(\frac{1}{2} \varrho|\boldsymbol{u}|^{2}+\varrho e(\varrho, \vartheta)+p(\varrho, \vartheta)\right) \boldsymbol{u}\right]=0, \\
& \partial_{t}(\varrho s(\varrho, \vartheta))+\operatorname{div}_{x}(\varrho s(\varrho, \vartheta) \boldsymbol{u}) \geq 0,
\end{aligned}
$$

where the pressure $p=p(\varrho, \vartheta)$, the specific internal energy $e=e(\varrho, \vartheta)$, and the specific entropy $s=s(\varrho, \vartheta)$ are interrelated through Gibbs' equation

$$
\vartheta D s(\varrho, \vartheta)=D e(\varrho, \vartheta)+p(\varrho, \vartheta) D\left(\frac{1}{\varrho}\right) .
$$

If $p, e, s$ comply with (1.5), then any smooth solution of (1.1)-(1.3) satisfies automatically the entropy balance in (1.4),

$$
\partial_{t}(\varrho s(\varrho, \vartheta))+\operatorname{div}_{x}(\varrho s(\varrho, \vartheta) \boldsymbol{u})=0 .
$$

This is no longer true for the weak solutions, here typically represented by shock waves, for which the entropy inequality (1.4) may be appended to the weak formulation of (1.1)(1.3) as an admissibility criterion imposed by the Second law of thermodynamics, see e.g. the monograph Benzoni-Gavage and Serre [3].

Our goal is to address the problem of weak (measure-valued)-strong uniqueness for the Euler system (1.1)-(1.4). Accordingly, we focus on identifying the largest class possible of measure-valued solutions, in which such a result holds, rather than the optimal one with respect to the expected regularity of solutions. To this end, we follow the approach advocated in [13], where equations (1.1), (1.2), with inequality (1.4), are supplemented with the total energy inequality

$$
\frac{\mathrm{d}}{\mathrm{d} t} \int_{\Omega}\left[\frac{1}{2} \varrho|\boldsymbol{u}|^{2}+\varrho e(\varrho, \vartheta)\right] \mathrm{d} x \leq 0,
$$

where $\Omega \subset R^{3}$ is the physical domain occupied by the fluid. To simplify presentation, we impose the periodic boundary conditions, meaning $\Omega$ can be identified with the flat torus

$$
\Omega=\left(\left.[0,1]\right|_{\{0,1\}}\right)^{3} .
$$

The problem is closed by prescribing the initial data 


$$
\varrho(0, \cdot)=\varrho_{0}, \vartheta(0, \cdot)=\vartheta_{0}, \boldsymbol{u}(0, \cdot)=\boldsymbol{u}_{0} .
$$

REMARK 1.1. We focus on the most difficult and physically relevant case of the spatial dimension $N=3$. The arguments can be easily adapted to $N=1,2$ as well.

The paper is organized as follows. In Section 2, we define the measure-valued solutions motivated by considering the cluster points of (hypothetical) families of weak solutions in the topology induced by the available a priori bounds. We point out that our class of measure-valued solutions is larger than that one proposed by Kröner and Zajaczkowski [19], where the entropy equality (1.6) is required. In particular, it includes all admissible weak solutions to the Euler system. Another conceptually new feature of our approach is that the dissipation defect "hidden" behind the inequality sign in (1.4) and (1.7) dominates the concentration error emerging in (1.2).

In Section 3, we establish and prove the main result of the present paper - the weak (measure-valued)-strong uniqueness principle. To this end, we use the relative energy inequality for system $(1.1),(1.2),(1.4),(1.7)$ identified in [14]. One of the principal difficulties is the hypothetical presence of vacuum zones on which the underlying equations fail to provide any control on the behavior of solutions. To avoid this problem, new phase variables must be considered - the density $\varrho$, the internal energy density $E=\varrho e$, and the momentum $\boldsymbol{m}=\varrho \boldsymbol{u}$.

Finally, possible extensions and applications of the main result are discussed in Section 4 .

\section{Measure-valued solutions.}

Motivated by [11], [18] we introduce the concept of dissipative measure-valued solution to the Euler system. For the sake of simplicity, we start with the constitutive equations of a perfect gas, specifically

$$
p(\varrho, \vartheta)=\varrho \vartheta, e(\varrho, \vartheta)=c_{\vartheta} \vartheta, s(\varrho, \vartheta)=\log \left(\frac{\vartheta^{c_{v}}}{\varrho}\right),
$$

where $c_{v}>0$ is the (constant) specific heat at constant volume.

In the seminal work of DiPerna [10], the measure-valued solutions have been identified as (weak) limits of weak solutions of system (1.1)-(1.4) or its suitable viscous approximation. In particular, all weak solutions of the problem should fall into the category of measure-valued solutions.

A weak solution $[\varrho, \vartheta, \boldsymbol{u}]$ of the Euler system in $(0, T) \times \Omega$, supplemented with the initial data (1.8), satisfies the family of integral identities:

$$
\int_{0}^{T} \int_{\Omega}\left[\varrho \partial_{t} \varphi+\varrho \boldsymbol{u} \cdot \nabla_{x} \varphi\right] \mathrm{d} x \mathrm{~d} t=-\int_{\Omega} \varrho_{0} \varphi(0, \cdot) \mathrm{d} x,
$$

for any $\varphi \in C_{c}^{\infty}([0, T) \times \Omega)$;

$$
\int_{0}^{T} \int_{\Omega}\left[\varrho \boldsymbol{u} \cdot \partial_{t} \boldsymbol{\varphi}+\varrho \boldsymbol{u} \otimes \boldsymbol{u}: \nabla_{x} \boldsymbol{\varphi}+\varrho \vartheta \operatorname{div}_{x} \boldsymbol{\varphi}\right] \mathrm{d} x \mathrm{~d} t=-\int_{\Omega} \varrho_{0} \boldsymbol{u}_{0} \cdot \boldsymbol{\varphi}(0, \cdot) \mathrm{d} x,
$$


for any $\varphi \in C_{c}^{\infty}\left([0, T) \times \Omega ; R^{3}\right)$;

$$
\begin{aligned}
& \int_{0}^{T} \int_{\Omega}\left[\left(\frac{1}{2} \varrho|\boldsymbol{u}|^{2}+c_{v} \varrho \vartheta\right) \partial_{t} \varphi+\left(\frac{1}{2} \varrho|\boldsymbol{u}|^{2}+c_{v} \varrho \vartheta\right) \boldsymbol{u} \cdot \nabla_{x} \varphi+\varrho \vartheta \boldsymbol{u} \cdot \nabla_{x} \varphi\right] \mathrm{d} x \mathrm{~d} t \\
& =-\int_{\Omega}\left(\frac{1}{2} \varrho_{0}\left|\boldsymbol{u}_{0}\right|^{2}+c_{v} \varrho_{0} \vartheta_{0}\right) \varphi(0, \cdot) \mathrm{d} x,
\end{aligned}
$$

for any $\varphi \in C_{c}^{\infty}([0, T) \times \Omega)$. In addition, a weak solution is called admissible if the entropy inequality is imposed

$$
\begin{aligned}
& \int_{0}^{T} \int_{\Omega}\left[\varrho Z\left(\log \left(\frac{\vartheta^{c_{v}}}{\varrho}\right)\right) \partial_{t} \varphi+\varrho Z\left(\log \left(\frac{\vartheta^{c_{v}}}{\varrho}\right)\right) \boldsymbol{u} \cdot \nabla_{x} \varphi\right] \mathrm{d} x \mathrm{~d} t \\
& \quad \leq-\int_{\Omega} \varrho_{0} Z\left(\log \left(\frac{\vartheta_{0}^{c_{v}}}{\varrho_{0}}\right)\right) \varphi(0, \cdot) \mathrm{d} x,
\end{aligned}
$$

for any $\varphi \in C_{c}^{\infty}([0, T) \times \Omega), \varphi \geq 0, Z \in B C(R), Z^{\prime} \geq 0$.

The weak solutions should also satisfy the natural constraints

$$
\varrho(t, x) \geq 0, \vartheta(t, x)>0 \text { a.a. in }(0, T) \times \Omega,
$$

in particular, the entropy density $\varrho s(\varrho, \vartheta)$ is well defined.

REMARK 2.1. The use of the cut-off function $Z$ in (2.4) is motivated by Chen and Frid [6]. Inequality (2.4) may be seen as a renormalized version of (1.4).

As is well known, smooth solutions of the Euler system may develop singularities in finite time for a fairly general class of initial data. The admissible weak solutions represent a physically grounded alternative providing the description of the system in an arbitrary time lap. Unfortunately, global-in-time existence of weak solutions is a largely open problem. In addition, the recent examples provided by the theory of convex integration, see Chiodaroli et al. [7], [8], show that uniqueness may fail in the multidimensional case even in the class of admissible weak solutions.

\subsection{Weak limits of weak solutions.}

In order to motivate our concept of measure-valued solution, we consider a family of initial data satisfying

$$
\begin{aligned}
& \varrho_{0, \varepsilon}>0, \quad \int_{\Omega} \varrho_{0, \varepsilon} \mathrm{d} x \geq M_{0}>0, \vartheta_{0, \varepsilon}>0, \log \left(\frac{\vartheta_{0, \varepsilon}^{c_{v}}}{\varrho_{0, \varepsilon}}\right) \geq s_{0}>-\infty, \\
& \int_{\Omega}\left[\frac{1}{2} \varrho_{0, \varepsilon}\left|\boldsymbol{u}_{0, \varepsilon}\right|^{2}+c_{v} \varrho_{0, \varepsilon} \vartheta_{0, \varepsilon}\right] \mathrm{d} x \leq e_{0},
\end{aligned}
$$

uniformly for $\varepsilon \rightarrow 0$. Suppose that $\left\{\varrho_{\varepsilon}, \vartheta_{\varepsilon}, \boldsymbol{u}_{\varepsilon}\right\}_{\varepsilon>0}$ are the corresponding weak solutions to the Euler system specified through (2.1)-(2.4). Our goal is to identify the cluster point of $\left\{\varrho_{\varepsilon}, \vartheta_{\varepsilon}, \boldsymbol{u}_{\varepsilon}\right\}_{\varepsilon>0}$ for $\varepsilon \rightarrow 0$. To this end, we first derive the available a priori bounds.

\subsubsection{A priori bounds.}

To begin, consider $Z \in B C(R)$, 


$$
Z^{\prime} \geq 0, Z(s) \begin{cases}<0 & \text { for } s<s_{0} \\ =0 & \text { for } s \geq s_{0}\end{cases}
$$

Take $\varphi=\psi(t), \psi \geq 0$ as a test function in the entropy inequality (2.4). Using (2.5) we deduce, after a straightforward manipulation, that

$$
\int_{\Omega} \varrho_{\varepsilon}(\tau, \cdot) Z\left(\log \left(\frac{\vartheta_{\varepsilon}^{c_{v}}(\tau, \cdot)}{\varrho_{\varepsilon}(\tau, \cdot)}\right)\right) \mathrm{d} x \geq 0 \text { for almost all } \tau \in(0, T) ;
$$

whence

$\log \left(\frac{\vartheta_{\varepsilon}^{c_{v}}}{\varrho_{\varepsilon}}\right) \geq s_{0}$ whenever $\varrho_{\varepsilon}>0 \Leftrightarrow \varrho_{\varepsilon} \leq \exp \left(-s_{0}\right) \vartheta_{\varepsilon}^{c_{v}}$ for almost all $(\tau, x) \in(0, T) \times \Omega$.

Similarly, we deduce from (2.3) and (2.5) that

$$
\int_{\Omega}\left[\frac{1}{2} \varrho_{\varepsilon}\left|\boldsymbol{u}_{\varepsilon}\right|^{2}+c_{v} \varrho_{\varepsilon} \vartheta_{\varepsilon}\right](\tau, \cdot) \mathrm{d} x \leq e_{0} \text { for almost all } \tau \in(0, T) .
$$

Next, in view of (2.6),

$$
\varrho_{\varepsilon}^{1+\left(1 / c_{v}\right)} \leq c\left(s_{0}\right) c_{v} \varrho_{\varepsilon} \vartheta_{\varepsilon}
$$

whence, in accordance with (2.7),

$$
\int_{\Omega} \varrho_{\varepsilon}^{1+\left(1 / c_{v}\right)}(\tau, \cdot) \mathrm{d} x \leq c\left(s_{0}, e_{0}\right) \text { for almost all } \tau \in(0, T) .
$$

By the same token,

$$
\varrho_{\varepsilon}\left|\log \left(\vartheta_{\varepsilon}\right)\right|^{q} \leq \begin{cases}c\left(s_{0}\right) \vartheta_{\varepsilon}^{c_{v}}\left|\log \left(\vartheta_{\varepsilon}\right)\right|^{q} \leq c\left(q, s_{0}\right) & \text { if } \vartheta_{\varepsilon} \leq 1 \\ \varrho_{\varepsilon} \vartheta_{\varepsilon} & \text { if } \vartheta_{\varepsilon} \geq 1\end{cases}
$$

therefore

$$
\int_{\Omega} \varrho_{\varepsilon}\left|\log \left(\vartheta_{\varepsilon}\right)\right|^{q}(\tau, \cdot) \mathrm{d} x \leq c\left(q, s_{0}, e_{0}\right), q \geq 1, \text { for almost all } \tau \in(0, T) .
$$

Finally, writing $\varrho_{\varepsilon} \boldsymbol{u}_{\varepsilon}=\sqrt{\varrho_{\varepsilon}} \sqrt{\varrho_{\varepsilon}} \boldsymbol{u}_{\varepsilon}$, we deduce from (2.7) and (2.8) that

$$
\int_{\Omega}\left|\varrho_{\varepsilon} \boldsymbol{u}_{\varepsilon}\right|^{p}(\tau, \cdot) \mathrm{d} x \leq c\left(s_{0}, e_{0}\right) \text { for almost all } \tau \in(0, T) \text { and some } p>1 \text {. }
$$

\subsubsection{Young measure.}

Unfortunately, the a priori bounds available are not strong enough to perform the pointwise limit in the nonlinearities in the weak formulation. Instead we use the characterization of limits of oscillatory sequences of functions via Young measures. Note that there is an additional problem as all bounds obtained in the previous section depend on $\varrho_{\varepsilon}$. In other words, we have no control over the behavior of $\boldsymbol{u}_{\varepsilon}, \vartheta_{\varepsilon}$ on the (hypothetical) vacuum zone. Consequently, it is more convenient to work with a new set of 
state variables - the density $\varrho$, the momentum $\boldsymbol{m}=\varrho \boldsymbol{u}$, and the internal energy density $E=c_{\vartheta} \varrho \vartheta$ - the norm of which is controlled at least in the Lebesgue space $L^{1}$.

Let

$$
\mathcal{F}=\left\{[\varrho, E, \boldsymbol{m}] \mid \varrho \in[0, \infty), E \in[0, \infty), \boldsymbol{m} \in R^{3}\right\}
$$

denote the new state space and let $L_{\text {weak- }(*)}^{\infty}((0, T) \times \Omega ; \mathcal{P}(\mathcal{F}))$ be the space of essentially bounded weakly-* measurable mappings $Y:(0, T) \times \Omega \rightarrow \mathcal{P}(\mathcal{F}),(t, x) \mapsto Y_{t, x}$.

By virtue of the fundamental theorem on Young measures, see e.g. Ball [1] , there exists a subsequence (not relabeled) of $\left\{\varrho_{\varepsilon}, E_{\varepsilon} \equiv c_{v} \varrho_{\varepsilon} \vartheta_{\varepsilon}, \boldsymbol{m}_{\varepsilon} \equiv \varrho_{\varepsilon} \boldsymbol{u}_{\varepsilon}\right\}_{\varepsilon>0}$ and a parameterized family of probability measures $\left\{Y_{t, x}\right\}_{(t, x) \in(0, T) \times \Omega}$,

$$
\left[(t, x) \mapsto Y_{t, x}\right] \in L_{\text {weak-(*) }}^{\infty}((0, T) \times \Omega ; \mathcal{P}(\mathcal{F}))
$$

such that

$$
\left\langle Y_{t, x} ; G(\varrho, E, \boldsymbol{m})\right\rangle=\overline{G(\varrho, E, \boldsymbol{m})}(t, x) \text { for any } G \in C_{c}(\mathcal{F}) \text { and a.a. }(t, x) \in(0, T) \times \Omega,
$$

whenever

$$
G\left(\varrho_{\varepsilon}, c_{v} \varrho_{\varepsilon} \vartheta_{\varepsilon}, \varrho_{\varepsilon} \boldsymbol{u}_{\varepsilon}\right) \rightarrow \overline{G(\varrho, E, \boldsymbol{m})} \text { weakly- }(*) \text { in } L^{\infty}((0, T) \times \Omega) .
$$

The parameterized family of measures $\left\{Y_{t, x}\right\}_{t, x \in(0, T) \times \Omega}$ is called Young measure associated to the sequence $\left\{\varrho_{\varepsilon}, c_{v} \varrho_{\varepsilon} \vartheta_{\varepsilon}, \varrho_{\varepsilon} \boldsymbol{u}_{\varepsilon}\right\}_{\varepsilon>0}$. As a consequence of $(2.6)$, we get

$$
\operatorname{supp}\left[Y_{t, x}\right] \subset\left\{[\varrho, E, \boldsymbol{m}] \in \mathcal{F} \mid \varrho^{1+c_{v}} \leq c_{v}^{-c_{v}} \exp \left(-s_{0}\right) E^{c_{v}}\right\} .
$$

As the nonlinearities appearing in the weak formulation do not in general belong to the class $C_{c}(\mathcal{F})$ (in the new set of variables $[\varrho, E, \boldsymbol{m}]$ ), validity of $(2.11)$ must be extended to a larger class of functions. If $G \in C(\mathcal{F})$ is such that

$$
\int_{0}^{T} \int_{\Omega}\left|G\left(\varrho_{\varepsilon}, c_{v} \varrho_{\varepsilon} \vartheta_{\varepsilon}, \varrho_{\varepsilon} \boldsymbol{u}_{\varepsilon}\right)\right| \mathrm{d} x \leq c \text { uniformly for } \varepsilon \rightarrow 0,
$$

then $G$ is $Y_{t, x}$ integrable for almost all $(t, x) \in(0, T) \times \Omega$ and

$$
\left[(t, x) \mapsto\left\langle Y_{t, x} ; G(\varrho, E, \boldsymbol{m})\right\rangle\right] \in L^{1}((0, T) \times \Omega) .
$$

The function $\left[(t, x) \mapsto\left\langle Y_{t, x} ; G(\varrho, E, \boldsymbol{m})\right\rangle\right]$ can be identified with the so-called biting limit of the family $\left\{G\left(\varrho_{\varepsilon}, c_{v} \varrho_{\varepsilon} \vartheta_{\varepsilon}, \varrho_{\varepsilon} \boldsymbol{u}_{\varepsilon}\right)\right\}_{\varepsilon>0}$, see Ball and Murat [2]. Finally, the same holds for any $G: \mathcal{F} \rightarrow R \cup\{\infty\}$ satisfying (2.12) and such that there exists a sequence $G_{m} \in C_{c}(\mathcal{F})$, $G_{m} \nearrow G$ in $\mathcal{F}$.

If (2.12) holds, we have

$$
G\left(\varrho_{\varepsilon}, c_{v} \varrho_{\varepsilon} \vartheta_{\varepsilon}, \varrho_{\varepsilon} \boldsymbol{u}_{\varepsilon}\right) \rightarrow \overline{G(\varrho, E, \boldsymbol{m})} \text { weakly- }(*) \text { in } \mathcal{M}([0, T] \times \Omega),
$$

for a suitable subsequence. Here, the singular part of the limit measure reflects possible concentrations in $\left\{\varrho_{\varepsilon}, \varrho_{\varepsilon} \vartheta_{\varepsilon}, \varrho_{\varepsilon} \boldsymbol{u}_{\varepsilon}\right\}_{\varepsilon>0}$. 
REMARK 2.2. Note carefully that the Young measure $\left[(t, x) \mapsto\left\langle Y_{t, x} ; G(\varrho, E, \boldsymbol{m})\right\rangle\right]$ is a parameterized family of non-negative measures acting on the phase space $\mathcal{F}$ while $\overline{G(\varrho, E, \boldsymbol{m})}$ is a signed measure on the physical space $[0, T] \times \Omega$.

The difference

$$
\mu_{G} \equiv \overline{G(\varrho, E, \boldsymbol{m})}-\left[(t, x) \mapsto\left\langle Y_{t, x} ; G(\varrho, E, \boldsymbol{m})\right\rangle\right] \in \mathcal{M}([0, T] \times \Omega),
$$

is called concentration defect measure. It vanishes whenever the family $\left\{G\left(\varrho_{\varepsilon}, c_{v} \varrho_{\varepsilon} \vartheta_{\varepsilon}, \varrho_{\varepsilon} \boldsymbol{u}_{\varepsilon}\right)\right\}_{\varepsilon>0}$ is equi-integrable (weakly precompact) in $L^{1}((0, T) \times \Omega)$.

We claim the following result proved in [11, Lemma 2.1].

LEMMA 2.3. Let

$$
|G(\varrho, E, \boldsymbol{m})| \leq F(\varrho, E, \boldsymbol{m}) \text { for all }(\varrho, E, \boldsymbol{m}) \in \mathcal{F} .
$$

Then

$\left|\overline{G(\varrho, E, \boldsymbol{m})}-\left\langle Y_{t, x} ; G(\varrho, E, \boldsymbol{m})\right\rangle\right| \leq \overline{F(\varrho, E, \boldsymbol{m})}-\left\langle Y_{t, x} ; F(\varrho, E, \boldsymbol{m})\right\rangle \equiv \mu_{F}$ in $\mathcal{M}([0, T] \times \Omega)$.

\subsubsection{The limit $\varepsilon \rightarrow \mathbf{0}$.}

We are ready to perform the limit for $\varepsilon \rightarrow 0$ in the weak formulation $(2.1)-(2.4)$.

STEP 1: In view of the uniform bounds (2.8) and (2.10), we can let $\varepsilon \rightarrow 0$ in (2.1) obtaining

$$
\int_{0}^{T} \int_{\Omega}\left[\left\langle Y_{t, x} ; \varrho\right\rangle \partial_{t} \varphi+\left\langle Y_{t, x} ; \boldsymbol{m}\right\rangle \cdot \nabla_{x} \varphi\right] \mathrm{d} x \mathrm{~d} t=-\int_{\Omega}\left\langle Y_{0, x} ; \varrho\right\rangle \varphi(0, \cdot) \mathrm{d} x,
$$

for any $\varphi \in C_{c}^{\infty}([0, T) \times \Omega)$, where $Y_{0, x}$ is the Young measure generated by the initial data. Note that, in accordance with (2.8) and (2.10), the families $\left\{\varrho_{\varepsilon}\right\}_{\varepsilon>0},\left\{\varrho_{\varepsilon} \boldsymbol{u}_{\varepsilon}\right\}_{\varepsilon>0}$ are equi-integrable and therefore concentrations do not occur. Finally, we deduce from (2.13) that

$$
\left[\int_{\Omega}\left\langle Y_{t, x} ; \varrho\right\rangle \varphi \mathrm{d} x\right]_{t=0}^{t=\tau}=\int_{0}^{\tau} \int_{\Omega}\left[\left\langle Y_{t, x} ; \varrho\right\rangle \partial_{t} \varphi+\left\langle Y_{t, x} ; \boldsymbol{m}\right\rangle \cdot \nabla_{x} \varphi\right] \mathrm{d} x \mathrm{~d} t
$$

for almost all $\tau \in(0, T)$ and for any $\varphi \in C^{1}([0, T] \times \Omega)$.

REMARK 2.4. Relation (2.14) can be justified for any $\tau \in[0, T)$, however, this is not needed for future analysis.

STEP 2: Keeping in mind the uniform energy bound (2.7), we consider $\varphi=\psi(t)$ as a test function in the total energy balance (2.3) obtaining

$$
\int_{0}^{T} \int_{\Omega}\left\langle Y_{t, x} ; \frac{1}{2} \frac{|\boldsymbol{m}|^{2}}{\varrho}+E\right\rangle \partial_{t} \psi \mathrm{d} x \mathrm{~d} t
$$




$$
\begin{aligned}
& +\int_{0}^{T}\left\langle\overline{\left(\frac{1}{2} \frac{|\boldsymbol{m}|^{2}}{\varrho}+E\right)}(t, \cdot) ; \Omega\right\rangle \partial_{t} \psi \mathrm{d} t-\int_{0}^{T} \int_{\Omega}\left\langle Y_{t, x} ; \frac{1}{2} \frac{|\boldsymbol{m}|^{2}}{\varrho}+E\right\rangle \partial_{t} \psi \mathrm{d} x \mathrm{~d} t \\
= & -\psi(0) \int_{\Omega}\left\langle Y_{0, x} ;\left(\frac{1}{2} \frac{|\boldsymbol{m}|^{2}}{\varrho}+E\right)\right\rangle \mathrm{d} x .
\end{aligned}
$$

REMARK 2.5. Note that the function

$$
[\varrho, \boldsymbol{m}] \in \operatorname{int}[\mathcal{F}] \mapsto \frac{|\boldsymbol{m}|^{2}}{\varrho},
$$

extended to be 0 whenever $\boldsymbol{m}=0$ and $\infty$ if $\varrho=0, \boldsymbol{m} \neq 0$ is a convex lower semicontinuous in $\mathcal{F}$; whence $Y_{t, x}$ measurable.

Thus we may infer that

$$
\left[\int_{\Omega}\left\langle Y_{t, x} ; \frac{1}{2} \frac{|\boldsymbol{m}|^{2}}{\varrho}+E\right\rangle \mathrm{d} x\right]_{t=0}^{t=\tau}+\mathcal{D}(\tau)=0,
$$

for almost all $\tau \in(0, T)$, with non-negative $\mathcal{D} \in L^{\infty}(0, T)$,

$$
\mathcal{D}(\tau)=\left\langle\overline{\left(\frac{1}{2} \frac{|\boldsymbol{m}|^{2}}{\varrho}+E\right)}(\tau, \cdot) ; \Omega\right\rangle-\int_{\Omega}\left\langle Y_{\tau, x} ; \frac{1}{2} \frac{|\boldsymbol{m}|^{2}}{\varrho}+E\right\rangle \mathrm{d} x
$$

for almost all $\tau \in(0, T)$. The quantity $\mathcal{D}$ will be termed dissipation defect.

REMARK 2.6. Recall that, in view of (2.7), we have

$$
\overline{\left(\frac{1}{2} \frac{|\boldsymbol{m}|^{2}}{\varrho}+E\right)} \in L^{\infty}(0, T ; \mathcal{M}(\Omega)) .
$$

REMARK 2.7. It follows from (2.15) that

$$
\operatorname{supp}\left[Y_{t, x}\right] \cap\{\varrho=0, \boldsymbol{m} \neq 0\}=\emptyset .
$$

In particular, if the measure $Y_{t, x}$ charges vacuum zone it must be only within the hyperplane $\boldsymbol{m}=0$.

STEP 3: Similarly, we deduce from the momentum equation (2.2) that

$$
\begin{aligned}
& {\left[\int_{\Omega}\left\langle Y_{t, x} ; \boldsymbol{m}\right\rangle \cdot \boldsymbol{\varphi} \mathrm{d} x\right]_{t=0}^{t=\tau}} \\
& \quad=\int_{0}^{\tau} \int_{\Omega}\left[\left\langle Y_{t, x} ; \boldsymbol{m}\right\rangle \cdot \partial_{t} \boldsymbol{\varphi}+\left\langle Y_{t, x} ; \frac{\boldsymbol{m} \otimes \boldsymbol{m}}{\varrho}\right\rangle: \nabla_{x} \boldsymbol{\varphi}+\left\langle Y_{t, x} ; p\right\rangle \operatorname{div}_{x} \boldsymbol{\varphi}\right] \mathrm{d} x \mathrm{~d} t \\
& \quad+\int_{0}^{\tau} \nabla_{x} \boldsymbol{\varphi}: \mathrm{d} \mu_{\mathcal{R}}
\end{aligned}
$$

for almost all $\tau \in(0, T)$ and for any $\varphi \in C^{1}\left([0, T] \times \Omega ; R^{3}\right)$, with the concentration error 


$$
\mu_{\mathcal{R}}=\overline{\left(\frac{\boldsymbol{m} \otimes \boldsymbol{m}}{\varrho}\right)}-\left\langle Y_{t, x} ; \frac{\boldsymbol{m} \otimes \boldsymbol{m}}{\varrho}\right\rangle+\bar{p} \mathbb{I}-\left\langle Y_{t, x} ; p\right\rangle \mathbb{I} \in L^{\infty}\left(0, T ; \mathcal{M}\left(\Omega ; R^{3 \times 3}\right)\right) .
$$

Note that $p=\left(1 / c_{v}\right) E$, and that, in view of Remark 2.7 , it is enough to set $\boldsymbol{m} \otimes \boldsymbol{m} / \varrho=0$ whenever $\boldsymbol{m}=0$.

In accordance with Lemma 2.3, we have an important relation between the concentration error $\mu_{\mathcal{R}}$ in (2.16) and the dissipation defect $\mathcal{D}$, namely

$$
\left|\int_{0}^{\tau} \nabla_{x} \varphi: \mathrm{d} \mu_{\mathcal{R}}\right| \leq\left\|\nabla_{x} \boldsymbol{\varphi}\right\|_{C([0, \tau] \times \Omega)} \int_{0}^{\tau} \mathcal{D}(t) \mathrm{d} t \text { for almost all } \tau \in(0, T) .
$$

SteP 4: Finally, the entropy balance (2.4) gives rise to

$$
\begin{aligned}
& {\left[\int_{\Omega}\left\langle Y_{t, x} ; \varrho Z(s)\right\rangle \varphi \mathrm{d} x\right]_{t=0}^{t=\tau}} \\
& \quad \geq \int_{0}^{\tau} \int_{\Omega}\left[\left\langle Y_{t, x} ; \varrho Z(s)\right\rangle \partial_{t} \varphi+\left\langle Y_{t, x} ; Z(s) \boldsymbol{m}\right\rangle \cdot \nabla_{x} \varphi\right] \mathrm{d} x \mathrm{~d} t
\end{aligned}
$$

for almost all $\tau \in(0, T)$, any $\varphi \in C^{1}([0, T] \times \Omega), \varphi \geq 0, Z \in B C(R), Z^{\prime} \geq 0$, where

$$
s=s(\varrho, E)=\log \left(\frac{E^{c_{v}}}{\varrho^{c_{v}+1}}\right) .
$$

The couple $\left\{Y_{t, x}, \mathcal{D}\right\}$ satisfying $(2.14),(2.15),(2.16),(2.17)$, and (2.18) represents a dissipative measure-valued solution of the complete Euler system (1.1)-(1.4).

\subsection{Dissipative measure-valued solutions.}

Motivated by the previous discussion, we introduce the concept of a dissipative measure-valued solution to the Euler system (1.1)-(1.4) for general constitutive relations. Although motivated by the preceding section, the measure-valued solutions introduced below represent an object formally independent of any approximation procedure, in particular they may not be a limit of a family of weak solutions.

In addition to Gibbs' equation (1.5), we assume the hypothesis of thermodynamic stability,

$$
\frac{\partial p(\varrho, \vartheta)}{\partial \varrho}>0, \frac{\partial e(\varrho, \vartheta)}{\partial \vartheta}>0 \text { for all } \varrho, \vartheta>0 .
$$

In particular, any function $G=G(\varrho, \vartheta, \boldsymbol{u})$ can be identified with a function of variables $[\varrho, E=\varrho e(\varrho, \vartheta), \boldsymbol{m}=\varrho \boldsymbol{u}]$ as

$$
G(\varrho, \vartheta, \boldsymbol{u})=G\left(\varrho, \vartheta(\varrho, E), \frac{\boldsymbol{m}}{\varrho}\right) \text { for all } \varrho>0, \vartheta>0, \boldsymbol{u} \in R^{3} .
$$

We simply write $G(\varrho, E, \boldsymbol{m})$ as the case may be.

REMARK 2.8. The former condition in (2.19) means that the compressibility of the gas is positive while the latter expresses positivity of the specific heat at constant 
volume. They may can be rephrased as convexity of the internal energy $e$ as a function

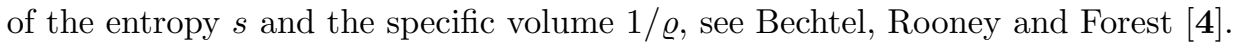

Definition 2.9 (Dissipative measure-valued solution). A family of probability measures $\left\{Y_{t, x}\right\}_{(t, x) \in(0, T) \times \Omega \text {, }}$

$$
(t, x) \mapsto Y_{t, x} \in L_{\text {weak-(*) }}^{\infty}((0, T) \times \Omega ; \mathcal{P}(\mathcal{F})),
$$

and the dissipation defect $\mathcal{D} \in L^{\infty}(0, T)$ represent a dissipative measure-valued solution of the Euler system (1.1)-(1.4) with the initial data $Y_{0, x}$ if:

(1)

$$
\left[\int_{\Omega}\left\langle Y_{t, x} ; \varrho\right\rangle \varphi \mathrm{d} x\right]_{t=0}^{t=\tau}=\int_{0}^{\tau} \int_{\Omega}\left[\left\langle Y_{t, x} ; \varrho\right\rangle \partial_{t} \varphi+\left\langle Y_{t, x} ; \boldsymbol{m}\right\rangle \cdot \nabla_{x} \varphi\right] \mathrm{d} x \mathrm{~d} t
$$

for almost all $\tau \in(0, T)$ and for any $\varphi \in C^{1}([0, T] \times \Omega)$;

(2)

$$
\begin{aligned}
& {\left[\int_{\Omega}\left\langle Y_{t, x} ; \boldsymbol{m}\right\rangle \cdot \boldsymbol{\varphi} \mathrm{d} x\right]_{t=0}^{t=\tau}} \\
& \quad=\int_{0}^{\tau} \int_{\Omega}\left[\left\langle Y_{t, x} ; \boldsymbol{m}\right\rangle \cdot \partial_{t} \boldsymbol{\varphi}+\left\langle Y_{t, x} ; \frac{\boldsymbol{m} \otimes \boldsymbol{m}}{\varrho}\right\rangle: \nabla_{x} \boldsymbol{\varphi}+\left\langle Y_{t, x} ; p(\varrho, E)\right\rangle \operatorname{div}{ }_{x} \boldsymbol{\varphi}\right] \mathrm{d} x \mathrm{~d} t \\
& \quad+\int_{0}^{\tau} \int_{\Omega}^{\tau} \nabla_{x} \boldsymbol{\varphi}: \mathrm{d} \mu_{\mathcal{R}},
\end{aligned}
$$

for almost all $\tau \in(0, T)$ and for any $\varphi \in C^{1}\left([0, T] \times \Omega ; R^{3}\right)$;

(3)

$$
\begin{aligned}
& {\left[\int_{\Omega}\left\langle Y_{t, x} ; \varrho Z(s(\rho, E))\right\rangle \varphi \mathrm{d} x\right]_{t=0}^{t=\tau}} \\
& \quad \geq \int_{0}^{\tau} \int_{\Omega}\left[\left\langle Y_{t, x} ; \varrho Z(s(\rho, E))\right\rangle \partial_{t} \varphi+\left\langle Y_{t, x} ; Z(s(\rho, E)) \boldsymbol{m}\right\rangle \cdot \nabla_{x} \varphi\right] \mathrm{d} x \mathrm{~d} t
\end{aligned}
$$

for almost all $\tau \in(0, T)$, any $\varphi \in C^{1}([0, T] \times \Omega), \varphi \geq 0, Z \in B C(R), Z^{\prime} \geq 0$;

(4)

$$
\left[\int_{\Omega}\left\langle Y_{t, x} ; \frac{1}{2} \frac{|\boldsymbol{m}|^{2}}{\varrho}+E\right\rangle \mathrm{d} x\right]_{t=0}^{t=\tau}+\mathcal{D}(\tau)=0
$$

where the dissipation defect $\mathcal{D}$ dominates the signed measure

$$
\mu_{\mathcal{R}} \in \mathcal{M}\left([0, T] \times \Omega ; R^{3 \times 3}\right),
$$

specifically,

$$
\left\|\mu_{\mathcal{R}}\right\|_{\mathcal{M}\left([0, \tau) \times \Omega ; R^{3 \times 3}\right)} \leq c \int_{0}^{\tau} \mathcal{D}(t) \mathrm{d} t
$$


for almost all $\tau \in(0, T)$.

As already pointed out, the dissipative measure-valued solutions are designed to retain the minimal piece of information inherited from the original Euler system in the course of some limit process. They may be seen as limits of families of weak solutions or their numerical approximations, cf. [11]. Notably, as shown in the next section, the dissipative measure-valued solutions comply with the weak-strong uniqueness principle. In this context, relation (2.24) plays the crucial role.

\section{Weak-strong uniqueness.}

Our ultimate goal is to show the main result of the present paper, namely, a dissipative measure-valued solution and a strong solution starting from the same initial data coincide as long as the latter exists. In addition to the natural physical principles encoded in (1.5), (2.19), we shall need a purely technical hypothesis

$$
|p(\varrho, \vartheta)| \lesssim(1+\varrho+\varrho e(\varrho, \vartheta)+\varrho|s(\varrho, \vartheta)|)
$$

Note that (3.1) is satisfied for a large family of gases for which $p \approx \varrho \vartheta$ including the perfect gas studied in Section 2. Here and hereafter, the symbol $a \lesssim b$ means $a \leq c b$ for a certain constant $c>0$.

\subsection{Relative energy.}

Let

$$
r \in C^{1}([0, T] \times \Omega), r>0, \Theta \in C^{1}([0, T] \times \Omega), \Theta>0, \boldsymbol{U} \in C^{1}\left([0, T] \times \Omega ; R^{3}\right),
$$

be given. Following [14], we introduce the ballistic free energy

$$
H_{\Theta}(\varrho, \vartheta)=\varrho e(\varrho, \vartheta)-\Theta \varrho s(\varrho, \vartheta)
$$

and the relative energy

$\mathcal{E}_{Z}(\varrho, \vartheta, \boldsymbol{u} \mid r, \Theta, \boldsymbol{U})=\frac{1}{2} \varrho|\boldsymbol{u}-\boldsymbol{U}|^{2}+\varrho e(\varrho, \vartheta)-\Theta \varrho Z(s(\varrho, \vartheta))-\frac{\partial H_{\Theta}(r, \Theta)}{\partial \varrho}(\varrho-r)-H_{\Theta}(r, \Theta)$.

The relative energy can be written in the new variables $[\varrho, E, \boldsymbol{m}]$ as

$\mathcal{E}_{Z}(\varrho, E, \boldsymbol{m} \mid r, \Theta, \boldsymbol{U})=\frac{1}{2} \varrho\left|\frac{\boldsymbol{m}}{\varrho}-\boldsymbol{U}\right|^{2}+E-\Theta \varrho Z(s(\varrho, E))-\frac{\partial H_{\Theta}(r, \Theta)}{\partial \varrho}(\varrho-r)-H_{\Theta}(r, \Theta)$.

In contrast with [14], the relative entropy functional depends also on the cut-off function appearing in the entropy inequality (2.22). The specific shape of $Z$ will be fixed below.

REMARK 3.1. Notation in (3.3) is slightly inconsistent as $\partial H_{\Theta}(r, \Theta) / \partial \varrho$ still denotes the derivative with respect to $\varrho$ of the function $H_{\Theta}(\varrho, \vartheta)$ considered in the "old" variables $(\varrho, \vartheta)$ rather than $(\varrho, E)$. We still believe this is convenient as the "test functions" $r$ and $\Theta$ are designed to mimick the density and the absolute temperature of the strong solution. 


\subsubsection{Relative energy inequality.}

Using the abstract formulation (2.20)-(2.23) we derive a functional relation

$$
\begin{aligned}
{\left[\int_{\Omega}\right.} & \left.\left\langle Y_{t, x} ; \mathcal{E}_{Z}(\varrho, E, \boldsymbol{m} \mid r, \Theta, \boldsymbol{U})\right\rangle \mathrm{d} x\right]_{t=0}^{t=\tau} \\
= & {\left[\int_{\Omega}\left\langle Y_{t, x} ; \frac{1}{2} \frac{|\boldsymbol{m}|^{2}}{\varrho}+E\right\rangle \mathrm{d} x\right]_{t=0}^{t=\tau}-\left[\int_{\Omega}\left\langle Y_{t, x} ; \boldsymbol{m}\right\rangle \cdot \boldsymbol{U} \mathrm{d} x\right]_{t=0}^{t=\tau} } \\
& +\left[\int_{\Omega}\left\langle Y_{t, x} ; \varrho\right\rangle\left(\frac{1}{2}|\boldsymbol{U}|^{2}-\frac{\partial H_{\Theta}(r, \Theta)}{\partial \varrho}\right) \mathrm{d} x\right]_{t=0}^{t=\tau}-\left[\int_{\Omega}\left\langle Y_{t, x} ; \varrho Z(s(\varrho, E))\right\rangle \Theta \mathrm{d} x\right]_{t=0}^{t=\tau} \\
& +\left[\int_{\Omega} \frac{\partial H_{\Theta}(r, \Theta)}{\partial \varrho} r-H_{\Theta}(r, \Theta) \mathrm{d} x\right]_{t=0}^{t=\tau} \\
= & -\mathcal{D}(\tau)-\left[\int_{\Omega}\left\langle Y_{t, x} ; \boldsymbol{m}\right\rangle \cdot \boldsymbol{U} \mathrm{d} x\right]_{t=0}^{t=\tau}+\left[\int_{\Omega}\left\langle Y_{t, x} ; \varrho\right\rangle\left(\frac{1}{2}|\boldsymbol{U}|^{2}-\frac{\partial H_{\Theta}(r, \Theta)}{\partial \varrho}\right) \mathrm{d} x\right]_{t=0}^{t=\tau} \\
& -\left[\int_{\Omega}\left\langle Y_{t, x} ; \varrho Z(s(\varrho, E))\right\rangle \Theta \mathrm{d} x\right]_{t=0}^{t=\tau}+\left[\int_{\Omega} \frac{\partial H_{\Theta}(r, \Theta)}{\partial \varrho} r-H_{\Theta}(r, \Theta) \mathrm{d} x\right]_{t=0}^{t=\tau} .
\end{aligned}
$$

Furthermore, using the entropy inequality (2.22), we get

$$
\begin{aligned}
{\left[\int_{\Omega}\right.} & \left.\left\langle Y_{t, x} ; \mathcal{E}_{Z}(\varrho, E, \boldsymbol{m} \mid r, \Theta, \boldsymbol{U})\right\rangle \mathrm{d} x\right]_{t=0}^{t=\tau}+\mathcal{D}(\tau) \\
\leq & -\int_{0}^{\tau} \int_{\Omega}\left[\left\langle Y_{t, x} ; \varrho Z(s(\varrho, E))\right\rangle \partial_{t} \Theta+\left\langle Y_{t, x} ; Z(s(\varrho, E)) \boldsymbol{m}\right\rangle \cdot \nabla_{x} \Theta\right] \mathrm{d} x \mathrm{~d} t \\
- & {\left[\int_{\Omega}\left\langle Y_{t, x}, \boldsymbol{m}\right\rangle \cdot \boldsymbol{U} \mathrm{d} x\right]_{t=0}^{t=\tau}+\left[\int_{\Omega}\left\langle Y_{t, x} ; \varrho\right\rangle\left(\frac{1}{2}|\boldsymbol{U}|^{2}-\frac{\partial H_{\Theta}(r, \Theta)}{\partial \varrho}\right) \mathrm{d} x\right]_{t=0}^{t=\tau} } \\
+ & {\left[\int_{\Omega} \frac{\partial H_{\Theta}(r, \Theta)}{\partial \varrho} r-H_{\Theta}(r, \Theta) \mathrm{d} x\right]_{t=0}^{t=\tau} . }
\end{aligned}
$$

The advantage of (3.4) is that all integrals on its right-hand side can be expressed by means of (2.20) and (2.21). Thus, repeating the arguments of [14, Section 3], we obtain

$$
\begin{aligned}
{\left[\int_{\Omega}\right.} & \left.\left\langle Y_{t, x} ; \mathcal{E}_{Z}(\varrho, E, \boldsymbol{m} \mid r, \Theta, \boldsymbol{U})\right\rangle \mathrm{d} x\right]_{t=0}^{t=\tau}+\mathcal{D}(\tau) \\
\leq & -\int_{0}^{\tau} \int_{\Omega}\left[\left\langle Y_{t, x} ; \varrho Z(s(\varrho, E))\right\rangle \partial_{t} \Theta+\left\langle Y_{t, x} ; Z(s(\varrho, E)) \boldsymbol{m}\right\rangle \cdot \nabla_{x} \Theta\right] \mathrm{d} x \mathrm{~d} t \\
& +\int_{0}^{\tau} \int_{\Omega}\left[\left\langle Y_{t, x} ; \varrho\right\rangle s(r, \Theta) \partial_{t} \Theta+\left\langle Y_{t, x} ; \boldsymbol{m}\right\rangle \cdot s(r, \Theta) \nabla_{x} \Theta\right] \mathrm{d} x \mathrm{~d} t \\
& +\int_{0}^{\tau} \int_{\Omega}\left[\left\langle Y_{t, x} ; \varrho \boldsymbol{U}-\boldsymbol{m}\right\rangle \cdot \partial_{t} \boldsymbol{U}+\left\langle Y_{t, x} ;(\varrho \boldsymbol{U}-\boldsymbol{m}) \otimes \frac{\boldsymbol{m}}{\varrho}\right\rangle: \nabla_{x} \boldsymbol{U}-\left\langle Y_{t, x} ; p(\varrho, E)\right\rangle\right. \\
& \left.\operatorname{div}_{x} \boldsymbol{U}\right] \mathrm{d} x \mathrm{~d} t+\int_{0}^{\tau} \int_{\Omega}\left[\left\langle Y_{t, x} ; r-\varrho\right\rangle \frac{1}{r} \partial_{t} p(r, \Theta)-\left\langle Y_{t, x} ; \boldsymbol{m}\right\rangle \cdot \frac{1}{r} \nabla_{x} p(r, \Theta)\right] \mathrm{d} x \mathrm{~d} t \\
& -\int_{0}^{\tau} \nabla_{x} \boldsymbol{U}: \mathrm{d} \mu_{\mathcal{R} .}
\end{aligned}
$$


The relation (3.5) holds for any dissipative measure-valued solution of the Euler system and any trio of smooth test functions satisfying (3.2). It can be seen as a measurevalued variant of the relative energy inequality derived in [14].

REMARK 3.2. The fact that (3.5) holds for any trio of "test functions" $[r, \Theta, \boldsymbol{U}]$ is important in view of possible future applications, cf. Section 4.

\subsection{Weak-strong uniqueness in the class of measure-valued solutions.}

Suppose that $[r, \Theta, \boldsymbol{U}]$ is a strong solution of the Euler system (1.1)-(1.3) starting from the initial data $\left[r_{0}, \Theta_{0}, \boldsymbol{U}_{0}\right]$ belonging to the class (3.2). We fix a compact set $K \subset$ $(0, \infty)^{2}$ containing the trajectories $\cup_{t \in[0, T], x \in \Omega}[r(t, x), \Theta(t, x)]$ and its image $\tilde{K} \subset(0, \infty)^{2}$ in the new phase variables

$$
(\varrho, \vartheta) \mapsto[\varrho, \varrho e(\varrho, \vartheta)]:(0, \infty)^{2} \rightarrow(0, \infty)^{2} .
$$

Finally, we consider a function $\Phi(\varrho, E)$,

$\Phi \in C_{c}^{\infty}(0, \infty)^{2}, 0 \leq \Phi \leq 1,\left.\Phi\right|_{\mathcal{U}}=1$, where $\mathcal{U}$ is an open neighborhood of $\tilde{K}$ in $(0, \infty)^{2}$.

For a measurable function $G(\varrho, E, \boldsymbol{m})$, we set

$$
G=G_{\mathrm{ess}}+G_{\mathrm{res}}, G_{\mathrm{ess}}=\Phi(\varrho, E) G(\varrho, E, \boldsymbol{m}), G_{\mathrm{res}}=(1-\Phi(\varrho, E)) G(\varrho, E, \boldsymbol{m}) .
$$

The idea, borrowed from [13], is that the "essential part" $G_{\text {ess }}$ describes the behavior of the non-linearity in the non-degenerate area where both $\varrho$ and $\vartheta$ are bounded below and above, while the "residual part" $G_{\text {res }}$ captures the behavior in the singular regime $\varrho, \vartheta \rightarrow 0$ or/and $\varrho, \vartheta \rightarrow \infty$.

Finally, we consider $Z=Z_{a, b} \in B C(R),-\infty \leq a<b \leq \infty$,

$$
Z_{a, b}(s)= \begin{cases}a & \text { for } s<a, \\ s & \text { for } s \in[a, b], \\ b & \text { for } s \geq b,\end{cases}
$$

and fix $a, b$ finite in such a way that

$$
\left[Z_{a, b}(s(\varrho, E))\right]_{\mathrm{ess}}=\Phi(\varrho, E) Z_{a, b}(s(\varrho, E))=\Phi(\varrho, E) s(\varrho, E)=[s(\varrho, E)]_{\mathrm{ess}} .
$$

\subsubsection{Initial data.}

We consider a dissipative measure-valued solution $\left\{Y_{t, x}, \mathcal{D}\right\}$ such that its initial value coincides with $\left[r_{0}, \Theta_{0}, \boldsymbol{U}_{0}\right]$, meaning

$$
Y_{0, x}=\delta_{\left[r_{0}(x), r_{0} e\left(r_{0}, \Theta_{0}\right)(x), r_{0} \boldsymbol{U}_{0}(x)\right]} \text { for almost all } x \in \Omega,
$$

where $\delta_{Y}$ denotes the Dirac distribution supported at $Y$. Accordingly,

$$
\int_{\Omega}\left\langle Y_{0, x} ; \mathcal{E}_{Z}\left(\varrho, E, \boldsymbol{m} \mid r_{0}(x), \Theta_{0}(x), \boldsymbol{U}_{0}(x)\right)\right\rangle \mathrm{d} x=0 .
$$


Taking $\varphi=1$ in (2.22) we get

$$
\int_{\Omega}\left\langle Y_{\tau, x} ; \varrho Z(s(\varrho, E))\right\rangle \mathrm{d} x \geq \int_{\Omega} r_{0} Z\left(s\left(r_{0}, \Theta_{0}\right)\right) \mathrm{d} x \text { for almost all } \tau \in(0, T) .
$$

As the initial data are regular, we deduce that there exists $a \in R$ such that

$$
\int_{\Omega}\left\langle Y_{\tau, x} ; \varrho Z(s(\varrho, E))\right\rangle \mathrm{d} x=0 \text { whenever } Z \leq 0, Z(s)=0 \text { for all } s \geq a .
$$

Consequently, we obtain

$$
\int_{\Omega}\left\langle Y_{\tau, x} ; \varrho Z_{a, b}(s(\varrho, E))\right\rangle \mathrm{d} x=\int_{\Omega}\left\langle Y_{\tau, x} ; \varrho Z_{-\infty, b}(s(\varrho, E))\right\rangle \mathrm{d} x,
$$

in particular

$$
\begin{aligned}
& -\int_{\Omega}\left\langle Y_{\tau, x} ; \varrho Z_{a, b}(s(\varrho, E))\right\rangle \mathrm{d} x \\
& \quad=-\int_{\Omega}\left\langle Y_{\tau, x} ; \varrho Z_{-\infty, b}(s(\varrho, E))\right\rangle \mathrm{d} x \geq-\int_{\Omega}\left\langle Y_{\tau, x} ; \varrho s(\varrho, E)\right\rangle \mathrm{d} x .
\end{aligned}
$$

Thus introducing a new relative energy

$$
\mathcal{E}(\varrho, E, \boldsymbol{m} \mid r, \Theta, \boldsymbol{U})=\frac{1}{2} \varrho\left|\frac{\boldsymbol{m}}{\varrho}-\boldsymbol{U}\right|^{2}+E-\Theta \varrho s(\rho, E)-\frac{\partial H_{\Theta}(r, \Theta)}{\partial \varrho}(\varrho-r)-H_{\Theta}(r, \Theta),
$$

and going back to (3.5), we obtain

$$
\begin{aligned}
\int_{\Omega} & \left\langle Y_{\tau, x} ; \mathcal{E}(\varrho, E, \boldsymbol{m} \mid r, \Theta, \boldsymbol{U})\right\rangle \mathrm{d} x+\mathcal{D}(\tau) \\
\leq & -\int_{0}^{\tau} \int_{\Omega}\left[\left\langle Y_{t, x} ; \varrho Z(s(\varrho, E))\right\rangle \partial_{t} \Theta+\left\langle Y_{t, x} ; Z(s(\varrho, E)) \boldsymbol{m}\right\rangle \cdot \nabla_{x} \Theta\right] \mathrm{d} x \mathrm{~d} t \\
& +\int_{0}^{\tau} \int_{\Omega}\left[\left\langle Y_{t, x} ; \varrho\right\rangle s(r, \Theta) \partial_{t} \Theta+\left\langle Y_{t, x} ; \boldsymbol{m}\right\rangle \cdot s(r, \Theta) \nabla_{x} \Theta\right] \mathrm{d} x \mathrm{~d} t \\
& +\int_{0}^{\tau} \int_{\Omega}\left[\left\langle Y_{t, x} ; \varrho \boldsymbol{U}-\boldsymbol{m}\right\rangle \cdot \partial_{t} \boldsymbol{U}+\left\langle Y_{t, x} ;(\varrho \boldsymbol{U}-\boldsymbol{m}) \otimes \frac{\boldsymbol{m}}{\varrho}\right\rangle: \nabla_{x} \boldsymbol{U}-\left\langle Y_{t, x} ; p(\varrho, E)\right\rangle\right. \\
& \left.\quad \operatorname{div}_{x} \boldsymbol{U}\right] \mathrm{d} x \mathrm{~d} t+\int_{0}^{\tau} \int_{\Omega}\left[\left\langle Y_{t, x} ; r-\varrho\right\rangle \frac{1}{r} \partial_{t} p(r, \Theta)-\left\langle Y_{t, x} ; \boldsymbol{m}\right\rangle \cdot \frac{1}{r} \nabla_{x} p(r, \Theta)\right] \mathrm{d} x \mathrm{~d} t \\
& -\int_{0}^{\tau} \nabla_{x} \boldsymbol{U}: \mathrm{d} \mu_{\mathcal{R}} \mathrm{d} t \text { for almost all } \tau \in(0, T),
\end{aligned}
$$

with some fixed $Z=Z_{a, b}, a<b$ finite.

\subsubsection{A Gronwall type argument.}

Our ultimate goal is to show that the right-hand side of (3.7) can be absorbed by the time average of the left-hand side. Thus, by means of the standard Gronwall argument, the left hand must vanish identically in $(0, T)$. To this end, we recall the coercivity properties of $\mathcal{E}$ proved in [13, Chapter 3, Proposition 3.2], 


$$
\begin{aligned}
& \mathcal{E}(\varrho, E, \boldsymbol{m} \mid r, \Theta, \boldsymbol{U}) \\
& \quad \gtrsim\left[|\varrho-r|^{2}+|E-r e(r, \Theta)|^{2}+\left|\frac{\boldsymbol{m}}{\varrho}-\boldsymbol{U}\right|^{2}\right]_{\mathrm{ess}}+\left[1+\varrho+\varrho|s(\varrho, E)|+E+\frac{|\boldsymbol{m}|^{2}}{\varrho}\right]_{\mathrm{res}} .
\end{aligned}
$$

SteP 1: We first use (2.24) to observe that

$$
\left|\int_{0}^{\tau} \nabla_{x} \boldsymbol{U}: \mathrm{d} \mu_{\mathcal{R}} \mathrm{d} t\right| \lesssim \int_{0}^{\tau} \mathcal{D}(t) \mathrm{d} t .
$$

Next, write

$$
\begin{aligned}
& \int_{\Omega}\left\langle Y_{t, x} ;(\varrho-\boldsymbol{m}) \otimes \frac{\boldsymbol{m}}{\varrho}\right\rangle: \nabla_{x} \boldsymbol{U} \mathrm{d} x \\
& \quad=\int_{\Omega}\left\langle Y_{t, x} ; \varrho \boldsymbol{U}-\boldsymbol{m}\right\rangle \otimes \boldsymbol{U}: \nabla_{x} \boldsymbol{U} \mathrm{d} x+\int_{\Omega}\left\langle Y_{t, x} ; \varrho\left(\boldsymbol{U}-\frac{\boldsymbol{m}}{\varrho}\right) \otimes\left(\frac{\boldsymbol{m}}{\varrho}-\boldsymbol{U}\right)\right\rangle: \nabla_{x} \boldsymbol{U} \mathrm{d} x
\end{aligned}
$$

where the right integral is controlled be the left-hand side of (3.7).

Consequently, as $[r, \Theta, \boldsymbol{U}]$ solve the Euler system (1.1)-(1.3), inequality (3.7) reduces to

$$
\begin{aligned}
& \int_{\Omega}\left\langle Y_{\tau, x} ; \mathcal{E}(\varrho, E, \boldsymbol{m} \mid r, \Theta, \boldsymbol{U})\right\rangle \mathrm{d} x+\mathcal{D}(\tau) \\
& \lesssim-\int_{0}^{\tau} \int_{\Omega}\left[\left\langle Y_{t, x} ; \varrho Z(s(\varrho, E))\right\rangle \partial_{t} \Theta+\left\langle Y_{t, x} ; Z(s(\varrho, E)) \boldsymbol{m}\right\rangle \cdot \nabla_{x} \Theta\right] \mathrm{d} x \mathrm{~d} t \\
&+\int_{0}^{\tau} \int_{\Omega}\left[\left\langle Y_{t, x} ; \varrho\right\rangle s(r, \Theta) \partial_{t} \Theta+\left\langle Y_{t, x} ; \boldsymbol{m}\right\rangle \cdot s(r, \Theta) \nabla_{x} \Theta\right] \mathrm{d} x \mathrm{~d} t \\
&+\int_{0}^{\tau} \int_{\Omega}\left[p(r, \Theta) \operatorname{div}_{x} \boldsymbol{U}-\left\langle Y_{t, x} ; p(\varrho, E)\right\rangle \operatorname{div}_{x} \boldsymbol{U}\right] \mathrm{d} x \mathrm{~d} t \\
&+\int_{0}^{\tau} \int_{\Omega}\left[\left\langle Y_{t, x} ; r-\varrho\right\rangle \frac{1}{r} \partial_{t} p(r, \Theta)-\left\langle Y_{t, x} ; \varrho \boldsymbol{U}\right\rangle \cdot \frac{1}{r} \nabla_{x} p(r, \Theta)-p(r, \Theta) \operatorname{div}_{x} \boldsymbol{U}\right] \mathrm{d} x \mathrm{~d} t \\
&+\int_{0}^{\tau}\left[\int_{\Omega}\left\langle Y_{t, x} ; \mathcal{E}(\varrho, E, \boldsymbol{m} \mid r, \Theta, \boldsymbol{U})\right\rangle \mathrm{d} x+\mathcal{D}(t)\right] \mathrm{d} t \text { for almost all } \tau \in(0, T) .
\end{aligned}
$$

STEP 2: Keeping in mind (3.6) we may rewrite

$$
\begin{aligned}
& -\int_{0}^{\tau} \int_{\Omega}\left[\left\langle Y_{t, x} ; \varrho Z(s(\varrho, E))\right\rangle \partial_{t} \Theta+\left\langle Y_{t, x} ; Z(s(\varrho, E)) \boldsymbol{m}\right\rangle \cdot \nabla_{x} \Theta\right] \mathrm{d} x \mathrm{~d} t \\
& \quad+\int_{0}^{\tau} \int_{\Omega}\left[\left\langle Y_{t, x} ; \varrho\right\rangle s(r, \Theta) \partial_{t} \Theta+\left\langle Y_{t, x} ; \boldsymbol{m}\right\rangle \cdot s(r, \Theta) \nabla_{x} \Theta\right] \mathrm{d} x \mathrm{~d} t \\
& =-\int_{0}^{\tau} \int_{\Omega}\left[\left\langle Y_{t, x} ;[\varrho Z(s(\varrho, E))]_{\mathrm{ess}}\right\rangle \partial_{t} \Theta+\left\langle Y_{t, x} ;[Z(s(\varrho, E)) \boldsymbol{m}]_{\mathrm{ess}}\right\rangle \cdot \nabla_{x} \Theta\right] \mathrm{d} x \mathrm{~d} t \\
& \quad+\int_{0}^{\tau} \int_{\Omega}\left[\left\langle Y_{t, x} ;[\varrho]_{\mathrm{ess}}\right\rangle s(r, \Theta) \partial_{t} \Theta+\left\langle Y_{t, x} ;[\boldsymbol{m}]_{\mathrm{ess}}\right\rangle \cdot s(r, \Theta) \nabla_{x} \Theta\right] \mathrm{d} x \mathrm{~d} t
\end{aligned}
$$




$$
\begin{aligned}
& -\int_{0}^{\tau} \int_{\Omega}\left[\left\langle Y_{t, x} ;[\varrho Z(s(\varrho, E))]_{\mathrm{res}}\right\rangle \partial_{t} \Theta+\left\langle Y_{t, x} ;[Z(s(\varrho, E)) \boldsymbol{m}]_{\mathrm{res}}\right\rangle \cdot \nabla_{x} \Theta\right] \mathrm{d} x \mathrm{~d} t \\
& +\int_{0}^{\tau} \int_{\Omega}\left[\left\langle Y_{t, x} ;[\varrho]_{\mathrm{res}}\right\rangle s(r, \Theta) \partial_{t} \Theta+\left\langle Y_{t, x} ;[\boldsymbol{m}]_{\mathrm{res}}\right\rangle \cdot s(r, \Theta) \nabla_{x} \Theta\right] \mathrm{d} x \mathrm{~d} t \\
= & \int_{0}^{\tau} \int_{\Omega}\left\langle Y_{t, x} ;[\varrho(s(r, \Theta)-s(\varrho, E))]_{\mathrm{ess}}\right\rangle \partial_{t} \Theta \mathrm{d} x \mathrm{~d} t \\
& +\int_{0}^{\tau} \int_{\Omega}\left\langle Y_{t, x} ;[\boldsymbol{m}(s(r, \Theta)-s(\varrho, E))]_{\mathrm{ess}}\right\rangle \cdot \nabla_{x} \Theta \mathrm{d} x \mathrm{~d} t \\
& -\int_{0}^{\tau} \int_{\Omega}\left[\left\langle Y_{t, x} ;[\varrho Z(s(\varrho, E))]_{\mathrm{res}}\right\rangle \partial_{t} \Theta+\left\langle Y_{t, x} ;[Z(s(\varrho, E)) \boldsymbol{m}]_{\mathrm{res}}\right\rangle \cdot \nabla_{x} \Theta\right] \mathrm{d} x \mathrm{~d} t \\
& +\int_{0}^{\tau} \int_{\Omega}\left[\left\langle Y_{t, x} ;[\varrho]_{\mathrm{res}}\right\rangle s(r, \Theta) \partial_{t} \Theta+\left\langle Y_{t, x} ;[\boldsymbol{m}]_{\mathrm{res}}\right\rangle \cdot s(r, \Theta) \nabla_{x} \Theta\right] \mathrm{d} x \mathrm{~d} t,
\end{aligned}
$$

where the residual terms are controlled in view of (3.8).

As for the essential components, we may pass to the original variables $(\varrho, \vartheta)$ to observe that

$$
[s(\varrho, \vartheta(\varrho, E))-s(r, \Theta)]_{\mathrm{ess}} \approx \frac{\partial s(r, \Theta)}{\partial \varrho}[\varrho-r]_{\mathrm{ess}}+\frac{\partial s(r, \Theta)}{\partial \vartheta}[\vartheta(\varrho, E)-\Theta]_{\mathrm{ess}},
$$

where the difference proportional to

$$
[\varrho-r]_{\mathrm{ess}}^{2}+[E-r e(r, \Theta)]_{\mathrm{ess}}^{2},
$$

is absorbed by the left-hand side of (3.9).

Summing up the previous discussion, we may replace (3.9) by

$$
\begin{aligned}
\int_{\Omega} & \left\langle Y_{\tau, x} ; \mathcal{E}(\varrho, E, \boldsymbol{m} \mid r, \Theta, \boldsymbol{U})\right\rangle \mathrm{d} x+\mathcal{D}(\tau) \\
\lesssim & -\int_{0}^{\tau} \int_{\Omega}\left\langle Y_{t, x} ; \varrho\left(\frac{\partial s(r, \Theta)}{\partial \varrho}[\varrho-r]_{\mathrm{ess}}+\frac{\partial s(r, \Theta)}{\partial \vartheta}[\vartheta(\varrho, E)-\Theta]_{\mathrm{ess}}\right)\right\rangle \partial_{t} \Theta \mathrm{d} x \mathrm{~d} t \\
& -\int_{0}^{\tau} \int_{\Omega}\left\langle Y_{t, x} ; \boldsymbol{m}\left(\frac{\partial s(r, \Theta)}{\partial \varrho}[\varrho-r]_{\mathrm{ess}}+\frac{\partial s(r, \Theta)}{\partial \vartheta}[\vartheta(\varrho, E)-\Theta]_{\mathrm{ess}}\right)\right\rangle \cdot \nabla_{x} \Theta \mathrm{d} x \mathrm{~d} t \\
& +\int_{0}^{\tau} \int_{\Omega}\left[p(r, \Theta) \operatorname{div}_{x} \boldsymbol{U}-\left\langle Y_{t, x} ; p(\varrho, E)\right\rangle \operatorname{div}_{x} \boldsymbol{U}\right] \mathrm{d} x \mathrm{~d} t \\
& +\int_{0}^{\tau} \int_{\Omega}\left[\left\langle Y_{t, x} ; r-\varrho\right\rangle \frac{1}{r} \partial_{t} p(r, \Theta)-\left\langle Y_{t, x} ; \varrho \boldsymbol{U}\right\rangle \cdot \frac{1}{r} \nabla_{x} p(r, \Theta)+\nabla_{x} p(r, \Theta) \cdot \boldsymbol{U}\right] \mathrm{d} x \mathrm{~d} t \\
& +\int_{0}^{\tau}\left[\int_{\Omega}\left\langle Y_{t, x} ; \mathcal{E}(\varrho, E, \boldsymbol{m} \mid r, \Theta, \boldsymbol{U})\right\rangle \mathrm{d} x+\mathcal{D}(t)\right] \mathrm{d} t \text { for almost all } \tau \in(0, T) .
\end{aligned}
$$

STEP 3: Using the fact that $r, \boldsymbol{U}$ satisfy the equation of continuity we get, after a tedious but straightforward manipulation, the identity

$$
(r-\varrho) \frac{1}{r} \partial_{t} p(r, \Theta)+\nabla_{x} p(r, \Theta) \cdot \boldsymbol{U}-\frac{\varrho}{r} \boldsymbol{U} \cdot \nabla_{x} p(r, \Theta)+\operatorname{div}_{x} \boldsymbol{U}(p(r, \Theta)-p(\varrho, \vartheta))
$$




$$
\begin{aligned}
= & \operatorname{div}_{x} \boldsymbol{U}\left(p(r, \Theta)-\frac{\partial p(r, \Theta)}{\partial \varrho}(r-\varrho)-\frac{\partial p(r, \Theta)}{\partial \vartheta}(\Theta-\vartheta)-p(\varrho, \vartheta)\right) \\
& +r(\varrho-r) \frac{\partial s(r, \Theta)}{\partial \varrho}\left(\partial_{t} \Theta+\boldsymbol{U} \cdot \nabla_{x} \Theta\right)+r(\vartheta-\Theta) \frac{\partial s(r, \Theta)}{\partial \vartheta}\left(\partial_{t} \Theta+\boldsymbol{U} \cdot \nabla_{x} \Theta\right)
\end{aligned}
$$

In view of hypothesis (3.1) the residual part of the expression on the left-hand side is controlled and we may go back to (3.10) to deduce the desired conclusion:

$$
\begin{aligned}
\int_{\Omega} & \left\langle Y_{\tau, x} ; \mathcal{E}(\varrho, E, \boldsymbol{m} \mid r, \Theta, \boldsymbol{U})\right\rangle \mathrm{d} x+\mathcal{D}(\tau) \\
\lesssim & \int_{0}^{\tau} \int_{\Omega}\left\langle Y_{t, x} ;\left[p(r, \Theta)-\frac{\partial p(r, \Theta)}{\partial \varrho}(r-\varrho)-\frac{\partial p(r, \Theta)}{\partial \vartheta}(\Theta-\vartheta(\varrho, E))-p(\varrho, \vartheta(\varrho, E))\right]_{\mathrm{ess}}\right\rangle \\
& \operatorname{div}_{x} \boldsymbol{U} \mathrm{d} x \mathrm{~d} t+\int_{0}^{\tau}\left[\int_{\Omega}\left\langle Y_{t, x} ; \mathcal{E}(\varrho, E, \boldsymbol{m} \mid r, \Theta, \boldsymbol{U})\right\rangle \mathrm{d} x+\mathcal{D}(t)\right] \mathrm{d} t \\
\lesssim & \int_{0}^{\tau} \int_{\Omega}\left\langle Y_{t, x} ;\left[|\varrho-r|^{2}+|E-r e(r, \Theta)|^{2}\right]_{\mathrm{ess}}\right\rangle \mathrm{d} x \mathrm{~d} t \\
& +\int_{0}^{\tau}\left[\int_{\Omega}\left\langle Y_{t, x} ; \mathcal{E}(\varrho, E, \boldsymbol{m} \mid r, \Theta, \boldsymbol{U})\right\rangle \mathrm{d} x+\mathcal{D}(t)\right] \mathrm{d} t \text { for almost all } \tau \in(0, T) .
\end{aligned}
$$

Applying Gronwall' lemma we deduce that the left-hand side of (3.11) vanishes for almost all $\tau \in(0, T)$.

We have shown the following result.

TheOREM 3.3 (Weak (measure-valued)-strong uniqueness principle). Let the thermodynamic functions $e=e(\varrho, \vartheta), s=s(\varrho, \vartheta)$, and $p=p(\varrho, \vartheta)$ satisfy Gibbs' relation (1.5), the hypothesis of thermodynamic stability (2.19), and let

$$
|p(\varrho, \vartheta)| \leq c(1+\varrho+\varrho|s(\varrho, \vartheta)|+\varrho e(\varrho, \vartheta)) .
$$

Let $[r, \Theta, \boldsymbol{U}]$ be a continuously differentiable classical solution of the Euler system (1.1)-(1.3) in $(0, T) \times \Omega$ starting from the initial data $\left(r_{0}, \Theta_{0}, \boldsymbol{U}_{0}\right)$ satisfying

$$
r_{0}>0, \Theta_{0}>0 .
$$

Assume that $\left[Y_{t, x} ; \mathcal{D}\right]$ is a dissipative measure-valued solution of the same problem in the sense specified in Definition 2.9 such that

$$
Y_{0, x}=\delta_{\left[r_{0}(x), r_{0} e\left(r_{0}, \Theta_{0}\right)(x), r_{0} \boldsymbol{U}_{0}(x)\right]} \text { for almost all } x \in \Omega .
$$

Then $\mathcal{D}=0$ and

$$
Y_{t, x}=\delta_{[r(t, x), r e(r, \Theta)(t, x), r \boldsymbol{U}(t, x)]} \text { for almost all }(t, x) \in(0, T) \times \Omega .
$$




\section{Conclusion.}

We have introduced the concept of dissipative measure-valued solution to the complete Euler system (1.1)-(1.4). Such a solution appears as a natural cluster point of families of weak solutions or their viscous approximations. We expect also certain numerical schemes to generate this kind of solutions, cf. [12]. The main result stated in Theorem 3.3 above asserts that a dissipative measure-valued solution coincides with a strong solution starting from the same initial data on the life span of the latter. In particular, if this is the case, any sequence generating the measure-valued solution necessarily converges pointwise to the strong solution. Such a result can be used for proving convergence of certain numerical schemes as in the simpler barotropic case discussed in $[\mathbf{1 2}]$.

The fact that the measure-valued formulation (2.20)-(2.24) contains the cut-off function $Z$ may seem restrictive although quite natural in the present context. The cut off can be dropped, meaning taking $Z(s)=s$, provided sufficiently strong a priori bounds are available to control integrability of $\varrho s \boldsymbol{u}=s \boldsymbol{m}$. As we have seen in Section 2 , these bounds followed from boundedness from below of the entropy of the system. Similar bounds can be obtained directly from the energy balance provided the constitutive thermodynamic functions satisfy a technical restriction

$$
\varrho|s(\varrho, \vartheta)|^{2} \lesssim(1+\varrho+\varrho e(\varrho, \vartheta)) .
$$

It can be shown that (4.1) holds for a general monoatomic gas satisfying the caloric equation of state

$$
p=\frac{2}{3} \varrho e,
$$

provided that the associated entropy $s=s(\varrho, \vartheta)$ complies with the Third law of thermodynamics, specifically,

$$
\lim _{\vartheta \rightarrow 0} s(\varrho, \vartheta)=0 \text { for any } \varrho>0,
$$

cf. [13, Chapter 1, Part 1.4].

\section{References}

[1] J. M. Ball, A version of the fundamental theorem for Young measures, In Lect. Notes in Physics, 344 (1989), 207-215.

[ 2 ] J. M. Ball and F. Murat, Remarks on Chacons biting lemma, Proc. Amer. Math. Soc., 107 (1989), 655-663.

[3] S. Benzoni-Gavage and D. Serre, Multidimensional hyperbolic partial differential equations, First order systems and applications, Oxford Mathematical Monographs, The Clarendon Press Oxford University Press, Oxford, 2007.

[4] S. E. Bechtel, F. J. Rooney and M. G. Forest, Connection between stability, convexity of internal energy, and the second law for compressible Newtonian fuids, J. Appl. Mech., 72 (2005), 299-300.

[5] Y. Brenier, C. De Lellis and L. Székelyhidi, Jr., Weak-strong uniqueness for measure-valued solutions, Comm. Math. Phys., 305 (2011), 351-361.

[6] G.-Q. Chen and H. Frid, Uniqueness and asymptotic stability of Riemann solutions for the com- 
pressible Euler equations, Trans. Amer. Math. Soc., 353 (2001), 1103-1117.

[ 7 ] E. Chiodaroli, A counterexample to well-posedness of entropy solutions to the compressible Euler system, J. Hyperbolic Differ. Equ., 11 (2014), 493-519.

[8] E. Chiodaroli, C. De Lellis and O. Kreml, Global ill-posedness of the isentropic system of gas dynamics, Comm. Pure Appl. Math., 68 (2015), 1157-1190.

[9] C. De Lellis and L. Székelyhidi, Jr., On admissibility criteria for weak solutions of the Euler equations, Arch. Rational Mech. Anal., 195 (2010), 225-260.

[10] R. J. DiPerna, Measure-valued solutions to conservation laws, Arch. Rational Mech. Anal., 88 (1985), 223-270.

[11] E. Feireisl, P. Gwiazda, A. Świerczewska-Gwiazda and E. Wiedemann, Dissipative measure-valued solutions to the compressible Navier-Stokes system, Calc. Var. Partial Differential Equations, 55 (2016), 141.

[12] E. Feireisl and M. Lukáčová-Medviďová, Convergence of a mixed finite element finite volume scheme for the isentropic Navier-Stokes system via dissipative measure-valued solutions, Found. Comput. Math., 18 (2018), 703-730.

[13] E. Feireisl and A. Novotný, Singular limits in thermodynamics of viscous fluids, Birkhäuser-Verlag, Basel, 2009.

[14] E. Feireisl and A. Novotný, Weak-strong uniqueness property for the full Navier-Stokes-Fourier system, Arch. Rational Mech. Anal., 204 (2012), 683-706.

[15] U. S. Fjordholm, R. Käppeli, S. Mishra and E. Tadmor, Construction of approximate entropy measure-valued solutions for hyperbolic systems of conservation laws, Found. Comput. Math., 17 (2017), 763-827.

[16] U. S. Fjordholm, S. Mishra and E. Tadmor, Arbitrarily high-order accurate entropy stable essentially nonoscillatory schemes for systems of conservation laws, SIAM J. Numer. Anal., 50 (2012), $544-573$.

[17] U. S. Fjordholm, S. Mishra and E. Tadmor, On the computation of measure-valued solutions, Acta Numer., 25 (2016), 567-679.

[18] P. Gwiazda, A. Świerczewska-Gwiazda and E. Wiedemann, Weak-strong uniqueness for measurevalued solutions of some compressible fluid models, Nonlinearity, 28 (2015), 3873-3890.

[19] D. Kröner and W. M. Zajaczkowski, Measure-valued solutions of the Euler equations for ideal compressible polytropic fluids, Math. Methods Appl. Sci., 19 (1996), 235-252.

[20] J. Málek, J. Nečas, M. Rokyta and M. Růžička, Weak and measure-valued solutions to evolutionary PDE's, Chapman and Hall, London, 1996.

\section{Jan BřEZINA}

Tokyo Institute of Technology

2-12-1 Ookayama, Meguro-ku

Tokyo 152-8550, Japan

E-mail: brezina@math.titech.ac.jp

\section{Eduard FEIREISL}

Institute of Mathematics of the Academy of Sciences of the Czech Republic

Žitná 25, CZ-115 67

Praha 1, Czech Republic

E-mail: feireisl@math.cas.cz 